\title{
Évaluation du potentiel de dix accessions de Jatropha curcas L. (Euphorbiaceae) au Niger
}

\author{
Zakari ABDOUL HABOU'1, Boubacar KATKORE¹, Tougiani ABASSE¹, François J. VERHEGGEN². \\ 1. Institut National de Recherche Agronomique du Niger, BP 429 Niamey (Niger), \\ 2. Unité d'Entomologie fonctionnelle et évolutive, Gembloux Agro-Bio Tech, Université de Liège, Passage des \\ Déportés 2, B-5030 Gembloux (Belgium), \\ E-mail adresse de correspondant : abdoulhabou_zakari@yahoo.fr*, Tel : 0022789565829.
}

Original submitted in on 25th October 2014. Published online at www.m.elewa.org on $31^{\text {st }}$ May 2014. http://dx.doi.org/10.4314/jab.v77i1.1

\section{RÉSUMÉ}

Objectif: Un essai de dix accessions de J. curcas a été installé au Centre Régional de Recherche Agronomique de Maradi au Niger, dans le but d'évaluer les performances des accessions de Jatropha curcas.

Methodologie et resultats: Chaque accession est représentée par une ligne de quinze pieds. II a été évalué, le taux de survie et les paramètres de croissances des individus mis en compétition. Les résultats obtenus montrent qu'il n'existe aucune différence significative entre les accessions testés sur la taille et leur diamètre au collet. Les accessions les mieux adaptées aux conditions du Niger sont celles d'origine malienne Katil 13 et GB 14 de Guinée Bisseau. Elles ont donnée un rendement de 511 et $445 \mathrm{~kg}$ par hectare respectivement en 2012, trois ans après leur semis. Les résultats de l'enquête montrent que $\mathrm{J}$. curcas a été introduit au Niger en 2004 par le biais de l'ICRISAT avec un essai de provenance de 16 accessions.

Conclusion et application des résultats: L'État s'est intéressé à cette culture par le lancement d'une plantation de pépinière à titre expérimentale de 150 ha à Guessalbodi. Les institutions de recherches nationales, les ONG et quelques privés se sont ensuite intéressés par la culture de J. curcas.

Mots-clés : Jatropha curcas, adaptation, rendement, Niger

\section{ABSTRACT}

Objective: A trial of the ten accessions for $J$ curcas was carried out at regional Agricultural Research Center of Maradi in Niger, with aim to evaluate the performance of Jatropha curcas accessions.

Methodology and results: Each accession was represented by two rows and each row has 15 plant trees of J. curcas. Data on the rate of survival and growth parameters of each tree were collected. Results revealed that there is no significant difference between accessions tested for height and diameter. Accessions Katil 13 and GB 14 respectively originated from Mali and Bisseau Guinea were best adapted for the Niger conditions. The yield value of 511 and $445 \mathrm{~kg}$ per hectare respectively obtained from Katil 13 and GB 14 in 2012. 
Conclusion and application of results: The Government of Niger has drawn attention to this crop and has launched a J. curcas plantation for seed production of 150 ha at Guessalbodi. National Research Institution. NGOs are also involved in J. curcas plantation for biofuel use.

Key words: Jatropha curcas adaptation, yield, Niger

\section{INTRODUCTION}

Les biocarburants sont présentés comme une alternative durable pour la résolution des problèmes (environnementale, économique et autres) liés à l'utilisation des carburants fossiles. Cependant le caractère durable de la production de biocarburant peut être mis à mal, si elle est réalisée de manière intensive: consommation de grandes quantités d'eau, pollution des eaux par l'usage d'engrais et de pesticides, épuisement des sols. C'est pour ces raisons que la production de biodiesel à partir de plantes oléagineuses telles que Jatropha curcas, plante assez rustique et très peu exigeante en eau et en intrants chimiques présente des meilleures perspectives. En raison des énormes potentialités que présente cette

\section{MATÉRIELS ET MÉTHODE}

Zone d'étude : Située à environ $670 \mathrm{~km}$ de Niamey, la région de Maradi s'étend sur une zone sahélosoudanienne caractérisée par un relief constitué d'un vaste plateau continental. La ville de Maradi est située au $13^{\circ} 30^{\prime} 3171 \mathrm{~N}$ et $7^{\circ} 06^{\prime} 0194 \mathrm{E}$.

Essai d'évaluation de performance de 10 accessions : L'essai expérimental sur les accessions de graines est installé dans le Centre Régional de Recherche Agronomique (CERRA-Maradi), qui est un démembrement de l'Institut National de Recherche Agronomique du Niger (INRAN). II est constitué de 10 accessions de J. curcas (Baas 38 (Inde); Baas 32 (Inde); Katil (Mali); Bfb1 (Burkina Faso); ISC14 (Inde) ;

\section{RÉSULTATS}

Toutes les accessions ont un taux de survie de 100\% sauf BAAS38 de provenance Indienne qui a $80 \%$ (Figure 1). L'analyse de variance montre qu'il n'existe aucune différence significative entre les accessions de $J$. curcas testés par rapport à la hauteur, le diamètre au collet des plants après trois ans le nombre de des plante les autorités maliennes ont lancé un Programme National de Valorisation Energétique de Pourghère (PNVEP). Un des objectifs de ce programme est l'accroissement de la production nationale de pourghère par l'aménagement de surfaces conséquentes en milieu rural. Cependant la vulgarisation de pourghère, plante jusque là utilisée essentiellement comme haie vive, se heurte à la méconnaissance des potentialités réelles des différents écotypes, en culture pure. $\mathrm{La}$ présente étude intitulée «Essai des accessions de Jatropha curcas » a pour objectif global d'évaluer les performances des accessions de Jatropha curcas.

SNES (Inde) 44 ; Falou (Mali) ; GB 14 (Guinée Bisseau); Las Pillas (Mali) 11 SENS 30 (Inde). Elles sont semées par semis direct le 6 Août 2009. Les graines sont fournies par ICRISAT. L'essai est constitué de 10 lignes dont chaque ligne représente une accession. Les plants sont espacés de 2,5 $\mathrm{m}$ sur les lignes et l'écartement entre les lignes est de $4 \mathrm{~m}$. les observations ont porté sur le taux de survie et les paramètres de croissance.

Analyse des données : Les données sont traitées par le logiciel Excel puis analysées par Minitab 16. Le test de Turkey au seuil de $5 \%$ a permis de faire la comparaison des moyennes.

feuilles. Par contre, les paramètres nombres de feuilles, la longueur, la largeur des feuilles et le rendement en graines ont montré des différences significatives $(p \leq 0,05)$. (Tableau 1). La hauteur moyenne des plants varie de 1,82 à $2 \mathrm{~m}$ et le diamètre au collet de 18 à 21 $\mathrm{cm}$. 


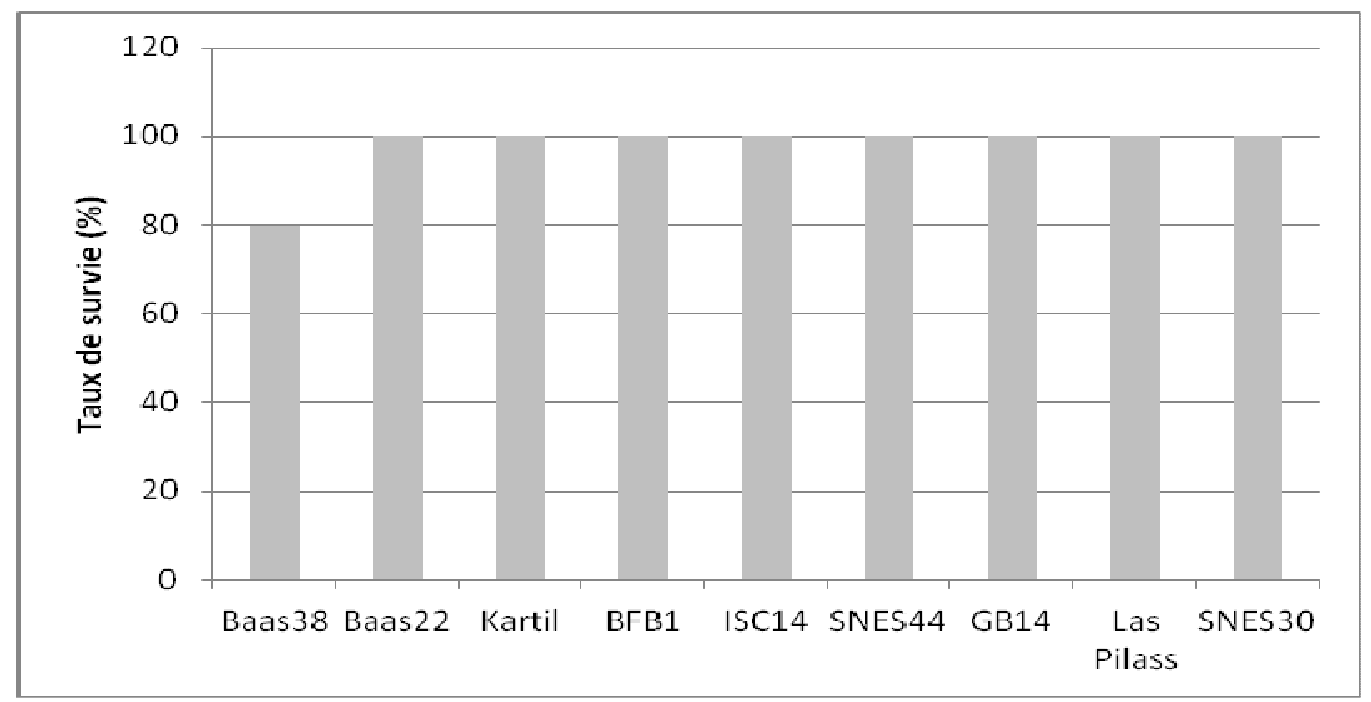

Figure 1: Taux de survie des accessions après trois ans d'adaptation.

Tableau 1 : paramètres de croissance des différentes accessions de $J$. curcas après trois ans d'installation sans entretien ni arrosage au Niger.

\begin{tabular}{|l|c|c|c|c|c|}
\hline Accessions & $\begin{array}{c}\text { Hauteur } \\
\mathbf{( c m})\end{array}$ & $\begin{array}{c}\text { Diamètre de tronc à } \\
\mathbf{5 0} \mathbf{~} \mathbf{( \mathbf { c m } )}\end{array}$ & Nombre de feuilles & $\begin{array}{c}\text { Longueur de } \\
\text { feuille } \mathbf{( c m})\end{array}$ & $\begin{array}{c}\text { Largeur de } \\
\text { feuille } \mathbf{( c m})\end{array}$ \\
\hline BFB1(17) & $1,90 \pm 0,40$ & $19,40 \pm 2,22$ & $44,67 \pm 14,20 \mathrm{ABC}$ & $8,85 \pm 0,79$ & $8,62 \pm 0,91$ \\
\hline GB (14) & $2,06 \pm 0,19$ & $21,29 \pm 2,10$ & $51,60 \pm 15,55 \mathrm{~A}$ & $8,67 \pm 0,66$ & $8,45 \pm 0,62$ \\
\hline BAAS38 (7) & $1,82 \pm 0,47$ & $18,12 \pm 3,30$ & $52,50 \pm 17,94 \mathrm{~A}$ & $9,38 \pm 0,56$ & $9,24 \pm 0,79$ \\
\hline BAAS22(10) & $1,91 \pm 0,40$ & $18,52 \pm 3,52$ & $48,07 \pm 20,82 \mathrm{AB}$ & $8,94 \pm 0,93$ & $8,77 \pm 1,03$ \\
\hline ISC 14(4) & $2,00 \pm 0,49$ & $20,18 \pm 3,49$ & $31,33 \pm 11,41 \mathrm{BCD}$ & $9,47 \pm 1,08$ & $9,55 \pm 1,27$ \\
\hline SNES44 (8) & $2,06 \pm 0,24$ & $21,11 \pm 2,22$ & $50,93 \pm 19,02 \mathrm{~A}$ & $9,39 \pm 0,97$ & $9,24 \pm 0,95$ \\
\hline SNES 30 (2) & $1,81 \pm 0,22$ & $19,27 \pm 2,53$ & $42,67 \pm 19,29 \mathrm{ABC}$ & $8,99 \pm 0,74$ & $9,00 \pm 0,67$ \\
\hline Falou (15) & $2,06 \pm 0,28$ & $21,08 \pm 2,97$ & $31,20 \pm 10,76 \mathrm{BCD}$ & $8,38 \pm 0,67$ & $8,15 \pm 0,73$ \\
\hline Katil (13) & $1,95 \pm 0,40$ & $18,77 \pm 2,38$ & $27,93 \pm 8,95 \mathrm{CD}$ & $8,43 \pm 0,74$ & $8,21 \pm 0,79$ \\
& & & & & \\
\hline Pillas (11) & $1,88 \pm 0,23$ & $19,03 \pm 2,48$ & $20,13 \pm 11,08 \mathrm{ABC}$ & $9,29 \pm 1,30$ & $8,61 \pm 1,22$ \\
\hline Probabilité & 0,29 & 0,01 & 0,00 & 0,001 & 0,000 \\
\hline
\end{tabular}

L'analyse de variance montre qu'il existe une différence significative entre les accessions par rapport au rendement en graines (Figure 2). L'accession la mieux adaptée aux conditions du Niger est celles d'origine malienne (Katil et GB 14 d'origine Guinéenne. Elles ont donnée un rendement de 511 et $445 \mathrm{~kg}$ par hectare respectivement en 2012. 


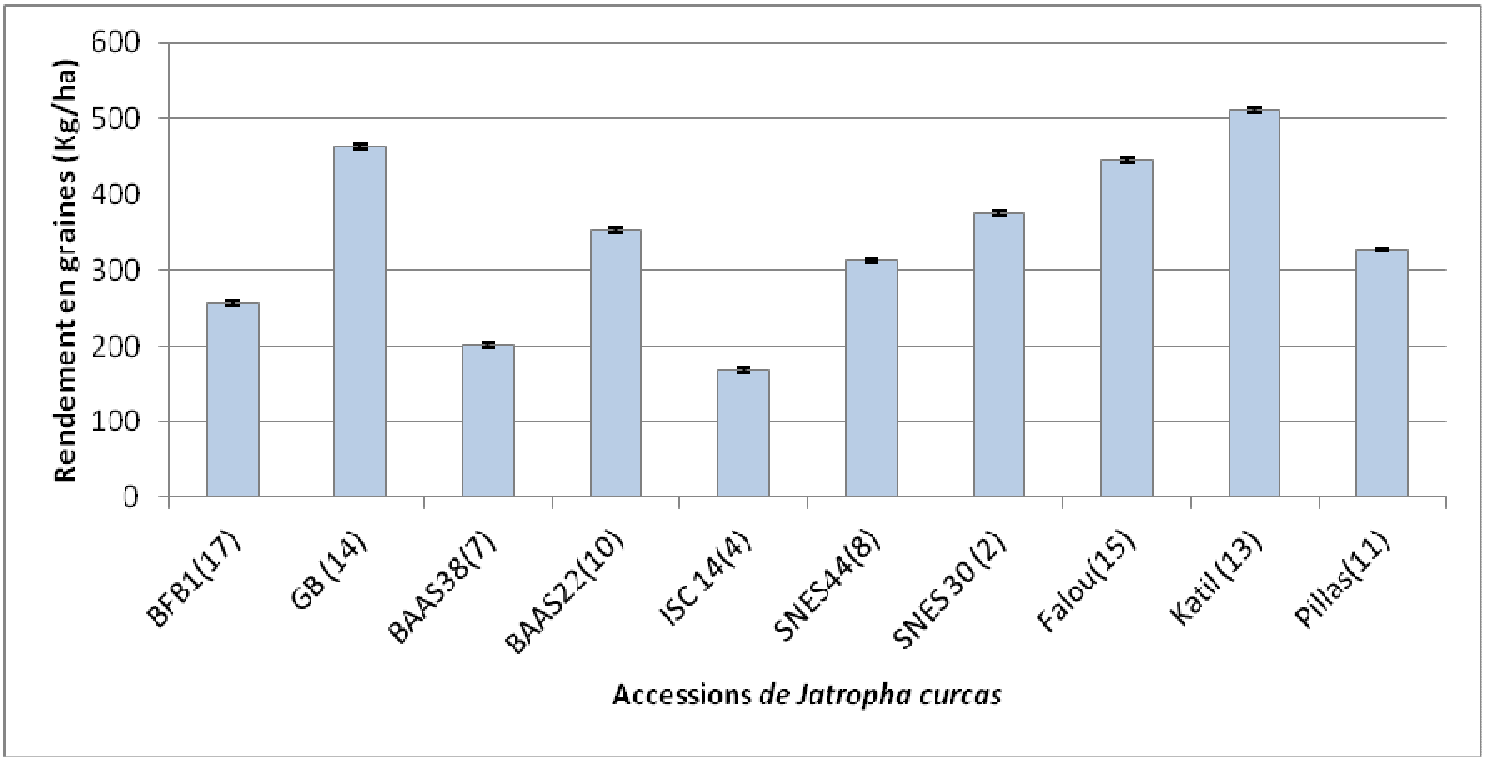

Figure 2 : Rendement en graines des accessions de Jatropha curcas au Niger obtenu en 2011.

\section{DISCUSSION}

Les résultats obtenus après l'évaluation du taux de survie ne montrent aucune variation en fonction de la durée des accessions. Les graines semées sont récoltées à la même période. Lorsque les graines sont nouvellement récoltées, elles ont un pourcentage élevé de germination. $J$. curcas est une plante oléagineuse contenant 43 à $60 \%$ d'huile (Neuwinger, 1994 ; Gandhi et al., 1995 ; Sharma et al.,1997 ; Wink et al., 1997 ; Gübitz et al., 1999 ; Beerens, 2007 ; Tewari, 2007). Cette huile végétale dont la composition et les caractéristiques varient en fonction des écotypes est très riche en triglycérides (Makkar et al., 1997 ; Gübitz et al., 1999 ; Achten et al., 2008). Ces triglycérides peuvent être hydrolysés par les triacylglycérols hydrolases dont les actions sont néfastes pour les graines en stockage car elles sont responsables de leur altération. Les caractères de croissances évalués montrent que les accessions diffèrent entre elles. Toutes les collections cultivées se sont bien comportées du point de vue croissance et développement. Ceci confirme la grande adaptabilité écologique de $\mathrm{J}$. curcas rapportée par plusieurs auteurs (Heller, 1996 ; Foidl et al., 1996 ; Makkar et Becker, 1999 ; Achten et al., 2008). En ce qui concerne la hauteur des arbustes, les accessions sont de même taille après trois d'adaptation. Le diamètre au collet est pratiquement le même pour toutes les accessions. BAAS38 et BAAS22 deux accessions d'origine indienne ont donné les plus grand nombre de feuille respectivement 52 et 51 . Elles présentent des feuilles les plus larges. Mais elles sont les moins productives et les plus tardives des accessions. Les données de trois ans obtenues entre 2007 et 2009 ont révélé des différences significatives entre les accessions $(P=0,036)$. Les deux meilleures accessions identifiées sont celles de la Guinée-Bissau et du Mali avec un rendement annuel moyen de plus que $320 \mathrm{~g}$ par arbre sur une durée de trois ans. Le rendement le plus bas a été donné par Las pilas, accession mexicaine (ICRISAT, 2009). Ces performances sont loin du rendement de $3-5 \mathrm{~kg}$ indiqué par de nombreux auteurs en tant que base acceptable pour les estimations de productivité de J. curcas. Même en conditions irriguées, le rendement est inférieur à 1,3 tha pour $96 \%$ des arbres (ICRISAT, 2009). L'ICRISAT effectue actuellement une étude de la variation morphologique et génétique qui servira de base pour l'amélioration génétique de Jatropha en conditions écologiques des tropiques semi-arides de l'Afrique de l'Ouest. Le climat très sec de la grande majorité du Niger ne permet pas de faire une production commerciale à grande échelle de J. curcas. Selon les données par Jongschaap et al. (2007), la production de graine de J. curcas est rentable dans des conditions d'humidité variant de 600 à plus de $1000 \mathrm{~mm}$ de pluie par an. En effet, dans les zones enregistrant une pluviométrie égale à $600 \mathrm{~mm}$, le projet Jatropha financé par Fondation FACT au Mali a eu des rendements de l'ordre de 0,3 tha-1. Des 
rendements de 1,5 et 6 tha $^{-1}$ sont obtenus dans les zones à 1000 et $1500 \mathrm{~mm}$ de pluie par an (FACT, 2010). La seule zone du Niger qui peut répondre à ses exigences est la zone soudanienne particulièrement le

\section{CONCLUSION}

Le potentiel d'adaptation de J. curcas a été évalué par le taux de sur vie et les paramètres de croissance des accessions après trois ans d'installation. Ainsi les deux accessions de provenance, indienne ont de taille haute mais produise moins des grains. Les écotypes pouvant

\section{RÉFÉRENCES}

Achten WMJ, Verchot L, Franken YJ, Mathijs E, Singh VP, Aerts R, Muys B. 2008. Jatropha biodiesel production and use. Biomass and Bioenergy, 32(12), 1063-1084

Achten W., MJ. ; Nielsen L., R.; Aerts, R.; Lengkeek, A., G.; Kjaer, E., D.; Trabucco, A.; Hansen J. K.; Maes W., H.; Graudal L.; Akinnifesi F. K. and Muys B., 2010. Towards domestication of Jatropha curcas. January 2010, Vol.1, No 1 , Pages 91-107, Futura Science, DOI 104155/bfs 09.4

Beerens P. 2007. Screw-pressing of Jatropha seeds for fuelling purposes in developed countries. MSc dissertation, Eindhoven University of Technology, Eindhoven, p. 63.

Danida., 2010. Domestication of Jatropha Curcas for Oil Production on Smallholder Farms in the Sudano-Sahelian Region, rapport, 2p. (http://ongoing-research.cgiar.org/factsheet)

FACT. 2006. Jatropha Handbook, First Draft, 45p

FACT., 2010. Jatropha curcas L.: from cultivate to application, $174 \mathrm{p}$

Foidl N, Foidl G, Sanchez M, Mittelbach M, Hackel S. 1996. Jatropha curcas $L$. as a source for the production of biofuel in Nicaragua. Biores. Tech., 58: 77-82.

Gandhi VM, Cherian KM, Mullky MJ. 1995. Toxicological studies on ratanjyot oil. Food Chem. Toxicol., 33: 39-42.

Gübitz GM, Mittelbach M, Trabi M. 1999. Exploitation of the tropical oil seed plant Jatropha curcas $\mathrm{L}$. Biores. Tech., 67: 73-82

Heller J. 1996. Physic Nut. Jatropha curcas L. Promoting the conservation and use of underutilized and neglected crops; Institute of Plant Genetics and Crop Plant Research département de Gaya où les isohyètes varient de 700 à $800 \mathrm{~mm}$ de pluie par an. La plantation de chez IBS Agro Industrie en est un exemple.

rentrer dans un programme d'amélioration et de sélection variétale en vue de mettre à la disposition des producteurs des nouveaux cultivars plus performants et plus précoces sont le cultivars de provenance Malienne Katil 13 et GB 14 de Guinée Bisseau.

(Gartersleben) and International Plant Genetic Resources Institute: Rome; Vol.1.

ICRISAT., 2009. Protégé la biodiversité, fournir des options, publication officielle de I'ICRISAT, 35 p.

Jongschaap R.E.E., Corré W., Bindraban P.S and Brandenburg W.A., 2007. Claims and Facts on Jatropha curcas L: Global Jatropha curcas evaluation, breeding and propagation program, Plant Research International.B.V, Wageningen, Report (158), 42p.

Makkar HPS, Becker K, Sporer F, Wink M. 1997. Studies on nutritive potential and toxic constituents of different provenances of Jatropha curcas. J. Agric. Food Chem., 45: 3152-3157.

Makkar HPS, Becker K. 1999. Plant toxins and detoxification methods to improve feed quality of tropical seeds Review. Asian-Aus. J. Anim. Sci., 12(3): 467-480.

Neuwinger HD. 1994. Afrikanische Arzneipflanzen und Jagdgifte. WV Gesmh, 450-457

R.N., 2011. Initiative " $3 \mathrm{~N}$ ». Pour la sécurité alimentaire et le Développement Agricole durable, 41p.

Sharma GD, Gupta SN, Khabiruddin M. 1997. Cultivation of Jatropha curcas as a future source of hydrocarbon and other industrial products. In Biofuels and Industrial Products from Jatropha curcas. Gübitz GM, Mittelbach M, Trabi M (eds). DBV Graz; 19-21.

Saadou M., 1998. Évaluation de la diversité biologique au Niger: Eléments constitutifs de la biodiversité végétale. Rapport du CNEDD, 138 $p$.

SALIFOU P.G., 2009. Comportement de différents écotypes de Jatropha curcas en zone 
Habou et al. J. Appl. Biosci. 2014. Évaluation du Potentiel de dix accessions de Jatropha Curcas L (Euphorbiaceae) au Niger

soudanienne du Mali, mémoire, IPR/IFRA de Katibougou, 46p ;

Wink M, Koschmieder C, Sauerwein M, Sporer F. 1997. Phorbol esters of J. curcas - biological activities and potential applications. In Biofuels and Industrial Products from Jatropha curcas. Gübitz GM, Mittelbach M, Trabi M (eds). DBV Graz; $160-166$ 